\title{
COUPLED SLIDING GRIDS AND ADAPTIVE GRID REFINEMENT FOR RANS SIMULATION OF AN OSCILLATING CAR MODEL
}

\author{
Emmanuel Guilmineau \\ LUNAM Université, CNRS \\ Laboratoire de recherche en Hydrodynamique \\ Energétique et Environnement Atmosphérique \\ UMR CNRS 6598 \\ 1 rue de la Noë, BP 92101 \\ 44321 Nantes Cedex 3, France \\ Email: Emmanuel.Guilmineau@ec-nantes.fr
}

\author{
Alban Leroyer \\ LUNAM Université, CNRS \\ Laboratoire de recherche en Hydrodynamique \\ Energétique et Environnement Atmosphérique \\ UMR CNRS 6598 \\ 1 rue de la Noë, BP 92101 \\ 44321 Nantes Cedex 3, France \\ Email: Alban.Leroyer@ec-nantes.fr
}

\author{
Patrick Queutey \\ LUNAM Université, CNRS \\ Laboratoire de recherche en Hydrodynamique \\ Energétique et Environnement Atmosphérique \\ UMR CNRS 6598 \\ 1 rue de la Noë, BP 92101 \\ 44321 Nantes Cedex 3, France \\ Email: Patrick.Queutey@ec-nantes.fr
}

\author{
Jeroen Wackers \\ LUNAM Université, CNRS \\ Laboratoire de recherche en Hydrodynamique \\ Energétique et Environnement Atmosphérique \\ UMR CNRS 6598 \\ 1 rue de la Noë, BP 92101 \\ 44321 Nantes Cedex 3, France \\ Email: Jeroen.Wackers@ec-nantes.fr
}

\begin{abstract}
Ground vehicles travelling on the road are often subjected to unsteady flows. The yaw angle increases with stronger wind conditions such as gusty cross winds caused by unsteady atmospheric conditions, tunnels, bridges or trucks passing. Separations appear on the leeward side of the body, interact with the base flow and cause unsteady vehicle loading. These aerodynamic elements, added to dynamic properties of the suspension, springing and tires are finally the factors of its dynamic stability and of the safe manoeuvrability appreciated by the driver. With an unsteady separation, it is difficult to have a mesh adapted to the flow. For improved accuracy, automatic adaptive grid refinement is a technique for optimising the grid in the simulation of fluid flow, by adapting the grid to the flow. This is done by locally dividing cells into smaller cells, or if necessary, by merging small cells backs into larger cells in order to undo earlier refinement.
\end{abstract}

The present work aims to explore the flow physics in which the vehicle is exposed to steady wind, but with continuous changes in the yaw angle. For this purpose, the unsteady numerical technique with a sliding grid approach is used in the flow solver ISIS-CFD, developed by the Laboratory of research in Hydrodynamics, Energetics, and Atmospheric Environment, exFluids Mechanics Laboratory of the Ecole Centrale de Nantes. The CFD simulation is carried out with the Explicit Algebraic Reynolds Stress Model (EARSM) turbulence model.

The numerical simulations are performed on the squareback Willy model. The Reynolds number is Re $=900,000$ and the yaw motion of the model is close to a sinusoidal motion with an amplitude of oscillation $\Delta \beta=10^{\circ}$ and a frequency $f=2 \mathrm{~Hz}$.

The numerical solution is compared with numerical results obtained in static positions. The pressure on the model is also compared with experimental data. 


\section{INTRODUCTION}

Ground vehicles traveling on the road are often subjected to unsteady flows. The yaw angle increases with stronger wind conditions such as gusty cross winds caused by unsteady atmospheric conditions, tunnels, bridges or trucks passing. Separations appear on the leeward side of the body, interact with the base flow and cause unsteady vehicle loading. These aerodynamic elements, added to dynamic properties of the suspension, springing and tires are finally the factors of its dynamic stability and of the safe manoeuvrability appreciated by the driver [1].

Unsteady flow conditions are much more difficult to simulate than steady flow conditions in the controlled conditions of a wind tunnel. The most common approach is to evaluate the aerodynamic performance using static conditions where, for example, the yaw angle of a vehicle or its relative position to another vehicle is changed discontinuously. The experiment or the simulation is then made for one position at a time. This approach is called quasi-steady or quasi-static as it is performed over a range of fixed positions of the vehicle, e.g. a fixed yaw angle. Although the positions from maximal positive to maximal negative yaw angle can be investigated with a small increment between two angle positions, this kind of investigation uses steady flow conditions and the resulting flow is different from the one in which continuous changes of yaw angle are made. These differences in the prediction of aerodynamic forces were observed by Garry and Cooper [2], who studied simple rectangular prisms rotating about their vertical axis in a uniform flow. They concluded that the difference in flows between steady and transient flow conditions is due to a lag in the response of the flow to continuous changes in the position of the vehicle when transient flow conditions are applied.

¿From a numerical point of view, this type of simulation requires a mesh with areas where a large number of points have been placed in advance in order to properly capture all the vortices. Another strategy is to have a mesh adapted to the flow. So, an automatic adaptive mesh is a good solution to follow the flow. Automatic adaptive grid refinement is a technique for optimising the grid in the simulation of fluid flow.

In order to study numerically an oscillating body, a first option is to oscillate the whole computational domain [3]. However this approach does not reflect the experimental setup. Another possibility is to deform the mesh in the vicinity of the model [4]. But again, the setup is not exactly the same as the experimental conditions as some cells outside the position of the turntable are deformed. The solution chosen for this study is to cut the mesh into two parts: a fixed part and a second corresponding to the plateau and will oscillate. The combination of these two meshes will be done by a sliding grid approach.

\section{WILLY MODEL}

The numerical simulations are performed on the square-back Willy model, which is similar to a van-type vehicle. A complete definition of the model is given by Guilmineau \& Chometon [5]. The overall length of the model is $\mathrm{L}=675 \mathrm{~mm}$, the width $\mathrm{W}$ $=240 \mathrm{~mm}$, the maximum height $\mathrm{H}=192 \mathrm{~mm}$ and its surface reference is the maximum cross section $\mathrm{S}_{r e f}=41791 \mathrm{~mm}^{2}$. The ground clearance is $\mathrm{G}=29 \mathrm{~mm}$ and the diameter of the four feet is $20 \mathrm{~mm}$, see Fig. 1 All other dimensions are defined by Guilmineau \& Chometon [5]. The origin of the Lilienthal axes $(\mathrm{X}, \mathrm{Y}, \mathrm{Z})$, linked to the model, lies at the point $\mathrm{O}$ located on the floor of the model. This point is also the centre of rotation of the model. Another coordinates system $(\mathrm{Xo}, \mathrm{Yo}, \mathrm{Z})$ is linked to the wind tunnel. The value of the yaw angle $\beta$ is positive when the right side of the model is to windward.

Experimental data are available for this model with an oscillating motion [3]. The Reynolds number based on the upstream velocity $V o$ and the length $L$ of the model is $\operatorname{Re}=0.9 \times 10^{6}$. The model in the experiments was placed on a turntable that allowed the model to oscillate with amplitude $\Delta \beta$ around the vertical axis. This turntable is actuated with a push-rod and a crankshaft [3]. The yaw angle versus the time is the physical root of

$$
a \tan ^{2}\left(\frac{\beta}{2}\right)+b \tan \left(\frac{\beta}{2}\right)+c=0
$$

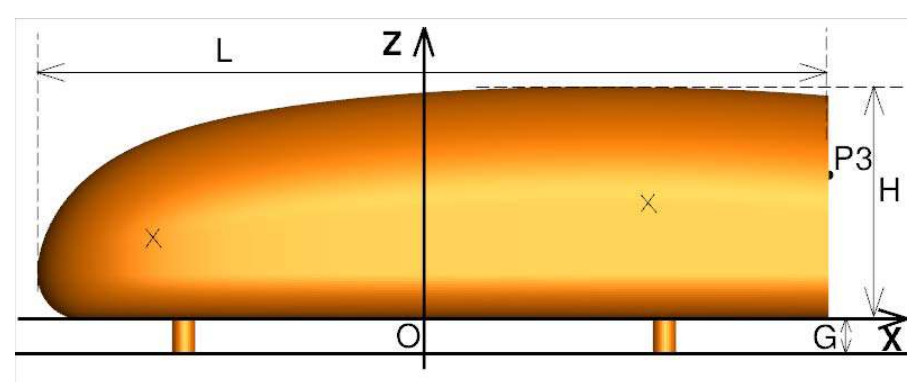

(a) SIDE VIEW.

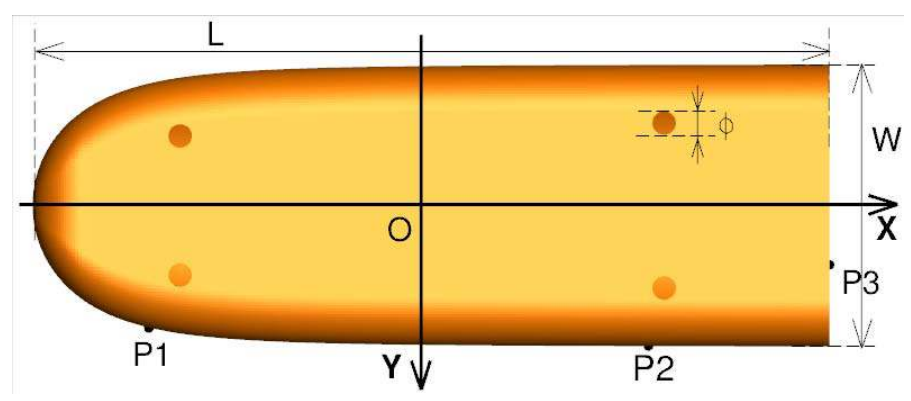

(b) BOTTOM VIEW.

FIGURE 1. WILLY DEFINITION. 
where

$$
\begin{gathered}
a=A+B\left(\cos \Delta \beta+\frac{\cos \alpha}{\sin \Delta \beta}\right) \\
b=B\left(\frac{2 l \sin \Delta \beta}{r}-2 \sin \Delta \beta \sin \alpha\right) \\
c=A-B(\cos \Delta \beta+\sin \Delta \beta \cos \alpha) \\
A=B(1+0.5 \sin (2 \Delta \beta) \cos \alpha)-2 r l \sin \alpha \\
B=\frac{2 r^{2}}{\sin ^{2} \Delta \beta}
\end{gathered}
$$

where $r=0.0255 \mathrm{~m}, l=0.3264 \mathrm{~m}$, and $\alpha=\omega \mathrm{t}$ where $\omega=2 \pi \mathrm{f}$ is an angular velocity and $t$ the time. For additional details of the experimental set-up and experimental equipment, we refer to Guilmineau \& Chometon [3]. In this paper, $\Delta \beta=10^{\circ}$ was simulated. Only the oscillation at a frequency of $\mathrm{f}=2 \mathrm{~Hz}$ corresponding to Strouhal number $S t=f L / U_{\infty}=0.068$ was investigated in the present study. Fig. 2 shows the evolution of the yaw angle versus the time. The choice of this frequency was made on the basis of the study of Watkins \& Saunders [6] who found that the range of frequencies of importance in cross-wind studies is 0.2 $2.0 \mathrm{~Hz}$, corresponding to $S t=0.09-0.9$.

\section{FLOW SOLVER DESCRIPTION \\ Governing Equations}

The ISIS-CFD flow solver, developed by the Numerical Modelling Group at the Fluid Mechanics Laboratory of the Ecole Centrale de Nantes, resolves the incompressible unsteady Reynolds-Averaged Navier-Stokes equations in two-fluid formulation. The conservation laws for momentum, total mass, and

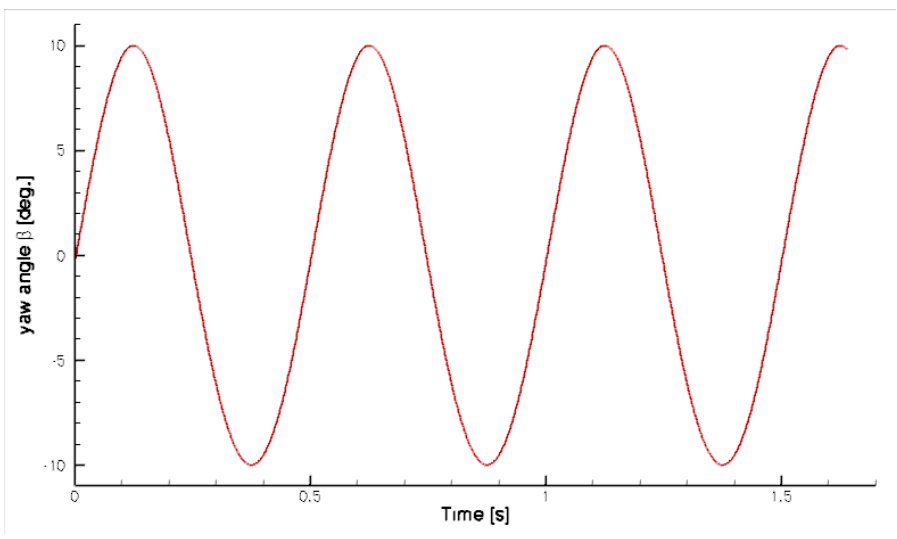

FIGURE 2. YAW ANGLE VERSUS TIME. mass of each fluid, are written as follows:

$$
\begin{gathered}
\frac{\delta}{\delta t} \int_{\mathscr{V}} \rho \vec{U} d V+\oint_{\mathscr{S}} \rho \vec{U}\left(\vec{U}-\overrightarrow{U_{d}}\right) \cdot \vec{n} d S= \\
\int_{\mathscr{V}}(\rho \vec{g}-\vec{\nabla} p) d V+\oint_{\mathscr{S}}\left(\mathbb{T}+\mathbb{T}_{t}\right) \cdot \vec{n} d S, \\
\oint_{\mathscr{S}} \vec{U} \cdot \vec{n} d S=0,
\end{gathered}
$$

where $\mathscr{V}$ is a volume, bounded by the closed surface $\mathscr{S}$ moving at the velocity $\vec{U}_{d}$ with a unit normal vector $\vec{n}$ directed outward. $\vec{U}$ and $p$ represent, respectively, the velocity and pressure fields. $\mathbb{T}$ and $\mathbb{T}_{t}$ refer to the viscous and Reynolds stress tensors, whereas $\vec{g}$ is the gravity vector. The time derivative following the moving grid is written $\delta / \delta t$. While $\mathbb{T}_{t}$ is determined according to the turbulence model used, $\mathbb{T}$ follows the classical relation of Newtonian fluid for incompressible flows. In the case of turbulent flows, additional transport equations for modelled variables are solved in a form similar to the momentum equations and they are discretized and solved using the same principles. Most closures based on a linear viscosity such as the $k-\omega$ SST model [7] are implemented. Two other more complex closures are also integrated in the flow solver ISIS-CFD: a model with the explicit algebraic Reynolds stress (EARSM) [8] and a Reynolds stress transport model (RSM) [9].

The ISIS-CFD flow solver can manage the threedimensional motions of an arbitrary number of non-deformable bodies but also deformable bodies with an imposed deformation. In this case, the body shape is imposed over time. The degrees of freedom (DOF) for each body can either be resolved, or be imposed by giving explicitly the law of variation of the parameter. The meshes are body-fitted grids, it is necessary to manage the motion, over time, of the nodes of the mesh representing each body [10]. In our case, we use a solid motion of the rotating mesh.

\section{Discretisation}

The flow equations of the previous subsection are discretised in a finite-volume framework. Pressure-velocity coupling is obtained through a Rhie \& Chow SIMPLE-type method [11]: in each time step, the velocity updates come from the momentum equations, Eqn. (7), and the pressure is given by the mass conservation law, Eqn. (8), transformed into a pressure equation.

The discretisation is face-based. While all unknown state variables are cell-centered, the systems of equations used in the implicit time stepping procedure are built face by face. Fluxes are computed in a loop over the faces and the contribution of each face is then added to the two cells next to the face. This technique poses no specific requirements on the topology of the cells. Therefore, the grids can be completely unstructured, cells with an arbitrary number of arbitrarily-shaped faces are accepted. 


\section{SLIDING GRID}

The primary objective of the sliding grid approach developed is to keep and to extend the actual capabilities of the flow solver to deal with non-matching sub-grids. The generalisation to multiple domains inside a single grid representation is done by colouring the cells, the nodes and the faces belonging to each sub-domain. A new boundary condition is introduced, called Non Matching Interface (NMI) on the physical boundary limiting the sub-domains. Although located on a physical boundary of a sub-domain, a coloured NMI face will be considered as a fluid face analogous to an inner face in the fluid or to a communication face on the boundary of a partition of the grid.

The sliding interfaces for fully unstructured grids are far more complex than for structured grid topologies. This is due to the explicit data structure required to address the connectivity, so that interpolation is not as relatively easy as it can be with the implicit addressing of a structural grid. As reported in the literature [12], various techniques have been developed to use sliding interfaces. Global flux conservation across the interface can be obtained but with constraints so that the flexibility to handle complex geometries is lost. In the context of the finite volume method with cell-centered schemes, it is possible to ensure a strict global mass conservation by replacing the faces of the control volumes by a set of a new faces built from the intersections of the sliding faces from each sub-domain sharing the sliding interface boundary. The main drawback is the limiting choice of the shape of the sliding boundary. It also results in a significant extra computational cost to build the new faces. Even for connecting dissimilar hexahedral meshes, the task is challenging in computational mechanics [13].

To compute the fluxes over the sliding interface, we need to establish connections between cells on the two sides of the interface. The procedure to connect these cells is performed at each time step in order to account for the rotation of the two subdomains with respect to each other. This procedure is chosen to remain as close as possible to what is done for standard cells. Thus, no specific interpolations are used. Instead, for a cell and face on the interface, we search the cell centre (in the other subdomain) which best matches the face.

The matching neighbour steps are searched in three steps, see Fig. 3.

1. A temporary ghost point is constructed on the outside of each sliding face. This point is the mirror image of the inside neighbour cell centre, except near sharp corners of the sliding interface where the normal vector to the face is used. Ghost points are constructed on each side of the interface, for the two sub-domains. The ghost points are not used for interpolation, only for the remainder of the search, see Fig. 3(a).

2. The current position of the sliding faces is gathered over all partitions to form a global table. Then, in each partition, a

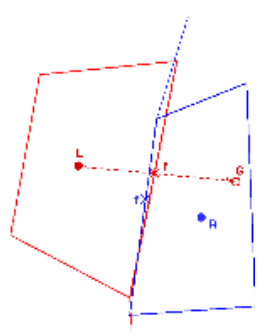

(a) CONSTRUCTION OF GHOST POINTS.

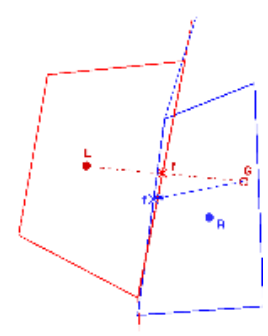

(b) SEARCHING THE GLOBAL FACES.

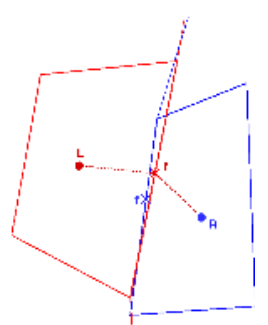
BOUR CELL. (c) NEW NEIGH-

FIGURE 3. SLIDING INTERFACES.

search algorithm is used to find the global sliding face closest to each local ghost point, see Fig. 3(b).

3. The inside neighbour cells of the faces found are used as outside neighbours for the local sliding faces. If the neighbour is on another processor, an MPI communication is established just like the one for the normal domain decomposition. If the two cells are on the same processor, the communication is performed locally. As opposed to the normal domain decomposition, a cell on a sliding interface may be a neighbour for more than one cell, or for none at all, see Fig. 3(c).

\section{GRID REFINEMENT PROCEDURE}

The grid refinement procedure developed for ISIS-CFD is integrated completely in the flow solver. The method is entirely parallelised, including automatic redistribution of grid over the processors.

During a flow computation, the refinement procedure is called repeatedly. In such a call, first the refinement criterion is calculated, then in a separate step of the procedure the grid is refined based on this criterion.

\section{Anisotropic refinement}

Anisotropic refinement is essential for our type of grid refinement. Isotropic refinement is very costly in three dimensions, since each refinement means a division in eight (for a hexahedron). Thus, creating very fine cells to accurately resolve a local flow phenomenon becomes almost impossible. However, by applying anisotropic refinement for flow features that need a fine grid in only one direction (for example a water surface), the total number of cells required can be greatly reduced or much finer flow details can be resolved.

Also, in unstructured hexahedral original grids, cells of completely different aspect ratios lie side by side. Therefore, when refining, we need to control the size of the fine cells in all their directions independently, otherwise refined grids may have smoothly varying sizes in one direction, but repeated changes 
from fine to coarse and back to fine in another. Isotropic refinement is not enough to prevent this. Therefore, directional refinement is the mandatory choice.

\section{Tensor refinement criteria}

For directional refinement, a way is needed to specify different cell sizes in different directions. The use of metric tensors as refinement criteria is such a way. This technique was first developed for the generation and refinement of unstructured tetrahedral meshes $[14,15]$. It is also an extremely useful and flexible framework for the refinement of unstructured hexahedral meshes.

For tensor-based refinement, the refinement criterion in each cell is a $3 \times 3$ symmetric positive definite matrix $C_{i}$. The refinement of the cells is decided as follows. Let the criterion tensors $C_{i}$ in each cell be known (they are computed from the flow solution). In each hexahedral cell, the cell size vectors $\mathbf{d}_{j, i}(\mathrm{j}=1 \cdots 3)$, which are the vectors between the opposing face centres in the three cell directions, are determined. Next, the modified sizes are computed as:

$$
\tilde{\mathbf{d}}_{j, i}=C_{i} \mathbf{d}_{j, i}
$$

Finally, a cell is refined in the direction $\mathrm{j}$ when the modified size exceeds a given, constant threshold value $T_{r}$ :

$$
\tilde{\mathbf{d}}_{j, i} \geq T_{r}
$$

\section{Refinement criteria}

The refinement criterion is an important part of the algorithm, as it controls the location of the refinement and the shape of the refined cells. The refinement criterion has to be carefully chosen depending on the flow problem that is simulated. In this paper, only the refinement criterion based on Hessian matrices is introduced. Other criteria, see [16], are available in the ISISCFD flow solver and used for hydrodynamic problems [17].

The Hessian matrix of second spatial derivatives of the solution is a common choice as a refinement criterion. We base this criterion on the pressure field, so it reacts to pressure induced flow features on the model, but not directly to the presence of boundary layers. The Hessian matrix is defined as

$$
\mathscr{H}_{i}=\left[\begin{array}{lll}
\left(p_{i}\right)_{x x} & \left(p_{i}\right)_{x y} & \left(p_{i}\right)_{x z} \\
\left(p_{i}\right)_{x y} & \left(p_{i}\right)_{y y} & \left(p_{i}\right)_{y z} \\
\left(p_{i}\right)_{x z} & \left(p_{i}\right)_{y z} & \left(p_{i}\right)_{z z}
\end{array}\right]
$$

The major difficulty in using the Hessian tensor as a refinement criterion is the accurate evaluation of the second derivatives, independent of the mesh. If the criterion computation is perturbed by local grid refinement, it may react more to existing grid refinement than to the pressure field. To prevent this undesired effect, numerical errors in the computed second derivatives must be significantly smaller than the derivatives themselves in all cells.

A particular problem, associated with unstructured hexahedral meshes, is that the grid remains irregular when it is refined. For structured grids, and even for most unstructured tetrahedral meshes, when the grid is refined the cells get more and more the same shape and size as their neighbours. On unstructured hexahedral meshes however, there will always be cells that are two times smaller than their direct neighbours. This means that numerical schemes which rely on mesh regularity to get good accuracy are not suited for these meshes; a useful scheme must give sufficient accuracy for arbitrary cell configurations.

For the computation of second derivatives, we use a leastsquares method based on third-order polynomials. In each cell, the polynomial is computed that best fits the pressure in the cell, its neighbours and its neighbours neighbours, in the least-squares sense. The approximated Hessian is constructed from the second derivatives of this polynomial. The least-squares procedure guarantees that the difference between the approximating polynomial and the real pressure is not in the space of third-order polynomials; therefore, it is at least fourth-order. Hence, the approximated second derivatives are second-order accurate, independent of the mesh geometry. For simpler methods like the well-known Gauss integration, this cannot be guaranteed.

To compute the refinement criterion, the Hessian is modified with a power law:

$$
\mathscr{C}_{H, i}=\left(\mathscr{H}_{i}\right)^{p}
$$

where $\left(\mathscr{H}_{i}\right)^{p}$ has the same eigenvectors as $\mathscr{H}_{i}$ and eigenvalues that are those of $\mathscr{H}_{i}$ (in absolute value) to the power $p$. In general, we use $p=\frac{1}{2}$.

\section{NUMERICAL SETUP}

The computational domain starts $4 \times \mathrm{L}$ in front of the model and extends to $5.4 \times \mathrm{L}$ behind the model. The width of the domain is $3 \times \mathrm{L}$ and the height is $1.5 \times \mathrm{L}$. The computational domain is represented in Fig. 4. The mesh is generated by the unstructured mesh generator Hexpress. This software generates meshes only containing hexaedrals. The mesh is composed of about 6.3 million nodes, 6.0 million cells with approximatively 94,500 faces on the model. The boundary conditions used for this computational domain are no slip walls for the Willy body geometry and a wall function for the floor of the computational domain, while the roof and the side walls of the computational domain are treated with Dirichlet conditions. At the inlet, the uniform velocity Vo is imposed while at the outlet, the pressure is imposed. Sliding interfaces are used at the end of the turntable. Thus, only the mesh 


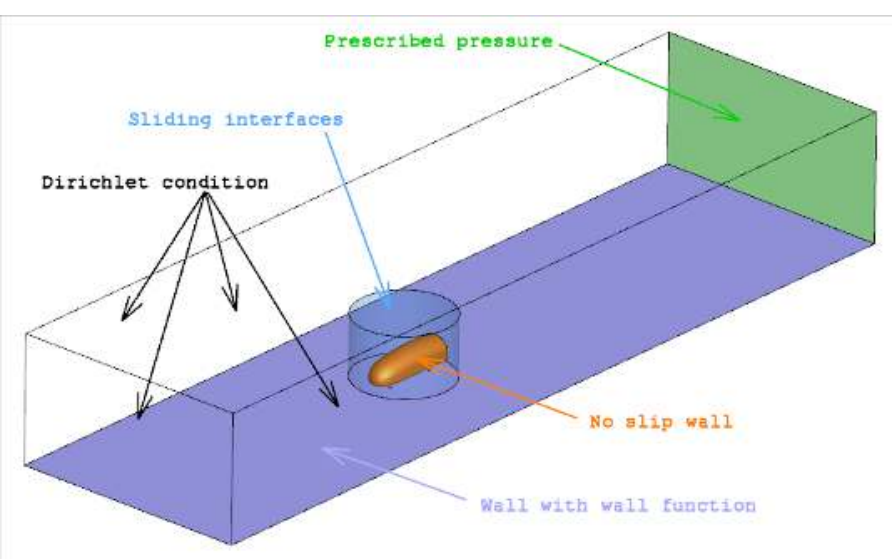

FIGURE 4. COMPUTATIONAL DOMAIN AND BOUNDARY CONDITIONS.

attached to the model oscillates, while the other part of the mesh remains fixed. With this approach the numerical model follows closely the experimental set-up, rather than the approach used in the previous numerical simulation [3] where the entire mesh had the same motion as the body.

The turbulence model used is the EARSM model which is the most reliable statistical turbulence modeling for predicting three-dimensional flows such as ship flows when intense longitudinal vortices spread out the aft part of the ship [18]. Usually, the CPU cost of this turbulence model is $30 \%$ higher than the $k-\omega$ turbulence model. The time step used for the simulation is $0.001 \mathrm{~s}$ that means 500 time steps are necessary to describe one oscillation period of the model.

For the simulations with a static position, the grid refinement procedure is called every 25 time steps while for the simulations with a dynamic position, this procedure is called every two time steps.

\section{RESULTS}

Before giving the results obtained when the car model oscillates, we present the numerical results for various yaw angles in static position.

\section{Static positions}

For the various static positions, we only rotate the mesh attached to the turntable.

The forces in the body reference frame are plotted versus the yaw angle $\beta$ in Fig. 5. The numerical results obtained with the initial mesh and the refined mesh are compared with experimental data. With the adaptive grid refinement, the number of added cells goes from 1.15 million cells at $\beta=0^{\circ}$ to 3.00 million cells at $\beta=10^{\circ}$. Thus for the yaw angle $\beta=0^{\circ}$, the number of cells is 7.15 millions and for the yaw angle $\beta=10^{\circ} 9.0$ millions. The

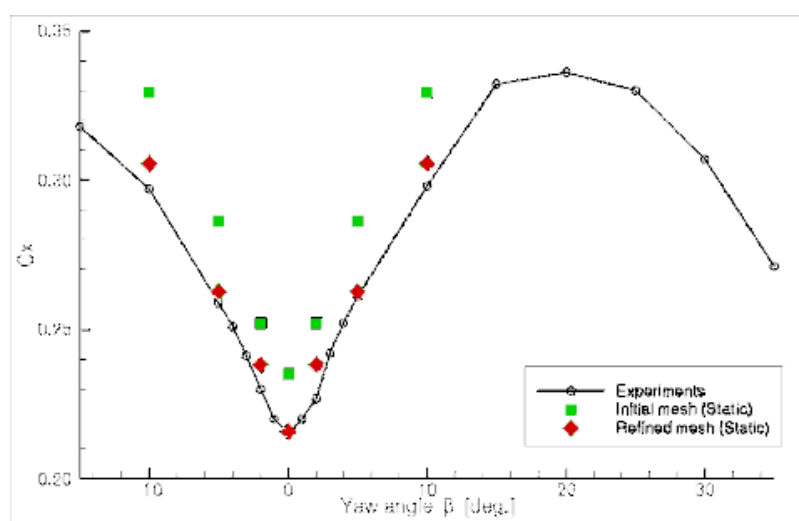

(a) DRAG COEFFICIENT.

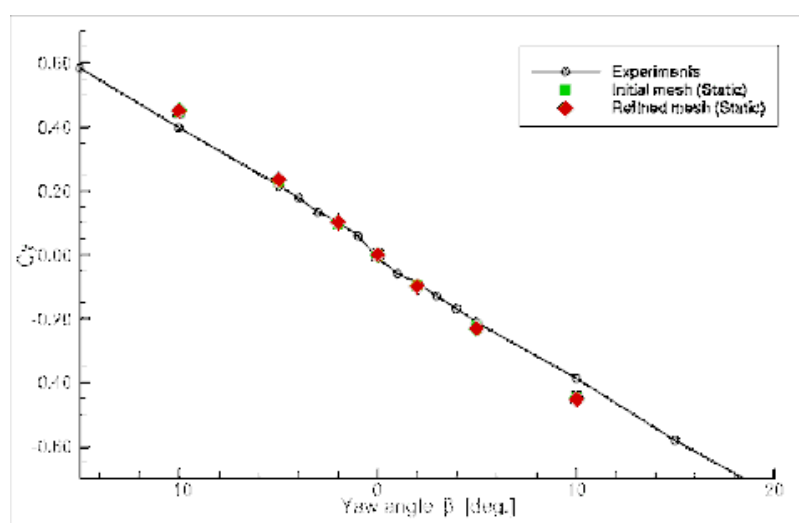

(b) SIDE FORCE COEFFICIENT.

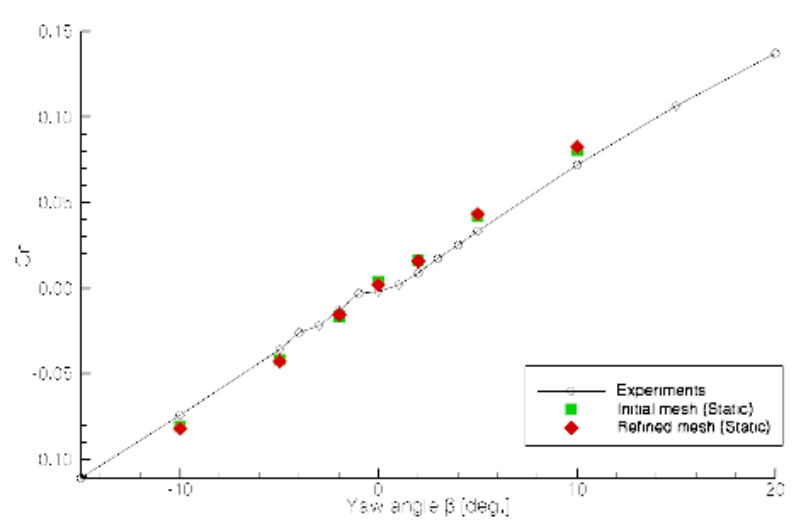

(c) YAWING MOMENT.

FIGURE 5. FORCE AND MOMENT COEFFICIENTS (STATIC POSITION).

drag coefficient is presented in Fig. 5(a). We can see that the results obtained with the adaptive grid refinement are in better agreement than the results obtained with the initial mesh. For all yaw angles, the numerical results obtained with the initial mesh overestimate the drag. The drag increases up to the yaw angle 
$\beta=20^{\circ}$. After this angle, the drag decreases. This behaviour is typical of a square-back model [1]. For the side force, see Fig. 5(b), and the yawing moment, see Fig. 5(c), the results of both simulations are very similar. The side force and the yawing moment rise linearly with the yaw angle. This linearity is a typical characteristic of real vehicles [1].

The difference of the drag coefficient between the simulation with and without automatic grid refinement can be clearly detected from a section-by-section analysis of the forces. The forces by section distributions in the Eiffel reference frame are obtained from the integration of the forces over the Willy model

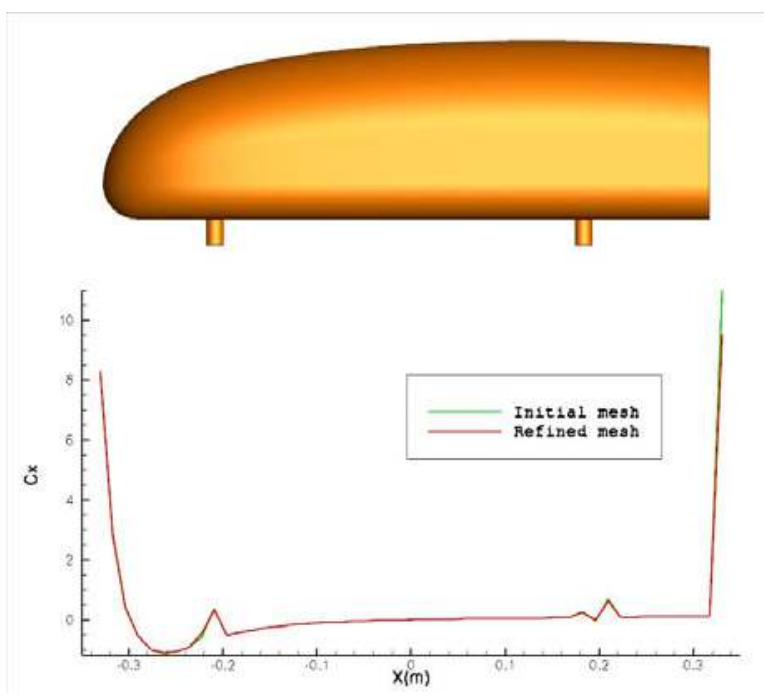

(a) $\beta=0^{\circ}$.
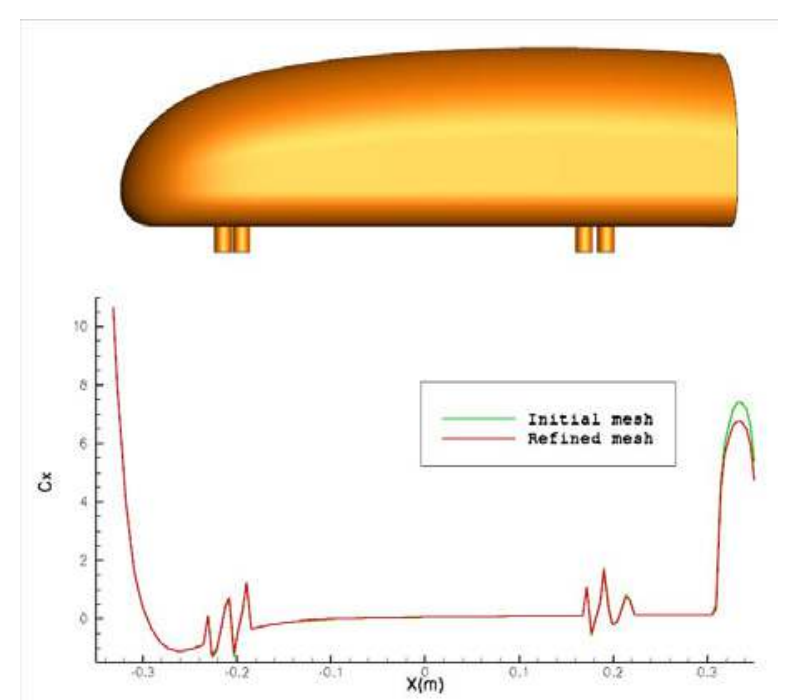

(b) $\beta=10^{\circ}$.

FIGURE 6. FORCE BY SECTION IN THE EIFFEL AXIS (STATIC POSITION). surface, divided in a number of sections. In this study, 150 equidistributed sections are used. Fig. 6 shows this distribution for two yaw angles. In both cases, the distribution is identical between the results obtained with the initial mesh and the results obtained with the refined mesh. Thus this drag difference is due to the base drag, as shown in Fig. 6(b) for X above 0.32.

\section{Dynamic position}

Fig. 7 shows the evolution of the number of mesh cells versus time. The evolution of the drag coefficient versus the time is also represented. After the first, transitional oscillation period, the signal becomes periodic. The number of cells varies from 6.34 million to 6.51 million. We notice that the number of cells is related to the evolution of the drag coefficient. When it is minimal, the number of cells is minimal and when $\mathrm{Cx}$ is maximal, the number of cells is maximal. The maximum number of cells is different from that obtained for the static calculations because the minimum size of the cells for the automatic grid refinement was higher in dynamic simulations than in static simulations.

Fig. 8 shows a comparison between the initial mesh and the refined mesh in the wake at X/L $=0.475$. The refinement procedure creates cells in the wake in the shear zones and where vortices exist.

The forces in the body axes are plotted versus the yaw angle $\beta$ in Figure 9. The results obtained in static position are also given. The drag coefficient is presented in Fig. 9(a). As for the static positions, the result obtained with the adaptive grid refinement is lower than the result obtained with the initial mesh. For the yaw angle $\beta=10^{\circ}$, the values obtained with the unsteady and the steady approach are similar. However, for the yaw angle $\beta=$ $0^{\circ}$, the drag obtained with the unsteady approach is larger than the drag obtained with the steady approach. The side force coefficient is given in Fig. 9(b) and the yawing moment in Fig. 9(c). Again, as for the static case, the differences between both simulations are low. In the prediction of forces, we observe fluctuations. These are probably due to the procedure of interpolation on the non-matching interface. The comparison with the previ-

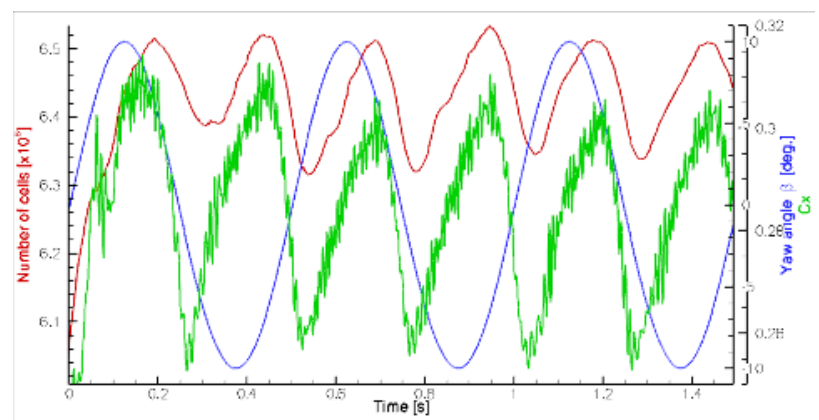

FIGURE 7. EVOLUTION OF THE NUMBER OF MESH CELLS. 


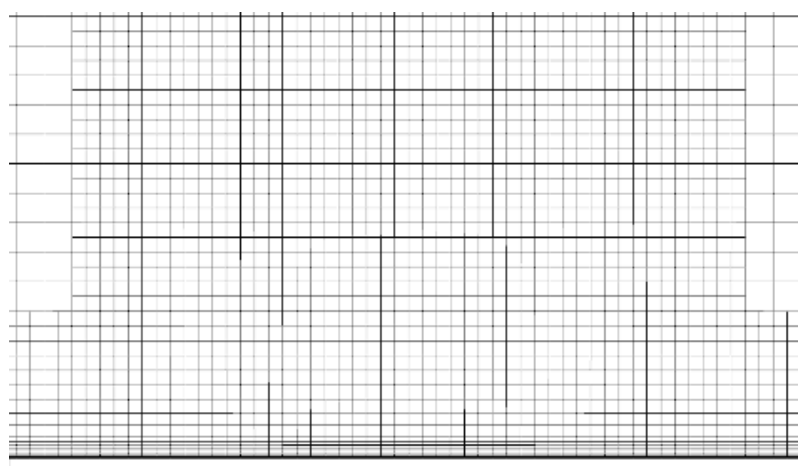

(a) INITIAL MESH.

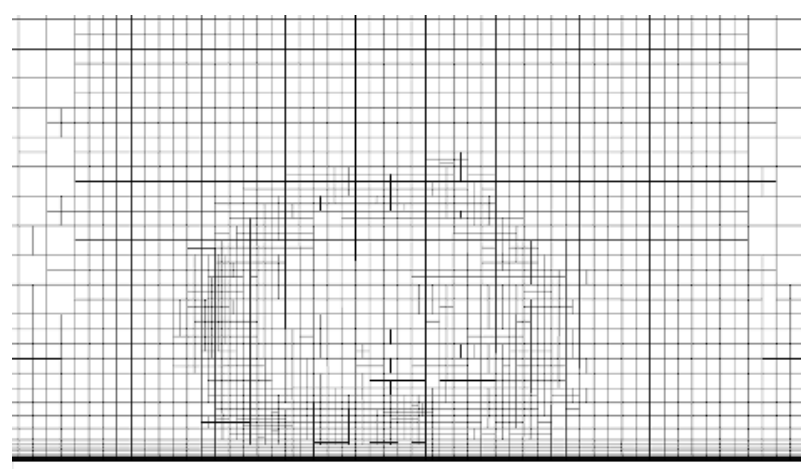

(b) REFINED MESH.

FIGURE 8. COMPARISON OF THE MESH AT Xo/L=0.475.

ous simulations [3] show that these results are very similar to those obtained with the refined mesh.

The comparison between the experimental and calculated wall pressure is given in Figure 10 for two pressure taps noted P1 and P2 located on the left side of the model and a third tap P3 located on its base. This pressure tap is not located in the symmetry plane of the model. The agreement of the numerical results with the experimental data is good for pressure taps P1 and P2. For the pressure $\mathrm{P} 3$, the numerical simulation produces higher $C_{P}$ values. This phenomenon is also observed with LES prediction [4]. For a "quasi-static" simulation, the pressure on the base is very difficult to obtain, even with a DES approach [19]. Thus, this can explain why the agreement for the pressure tap P3 is not so good. The pressure for these three pressure taps is also compared with the numerical results obtained in the previous simulation [3]. For the both tap located on the side of the model, the results are very similar. For the tap located on the base of the model, the result is different from those obtained with the sliding grid approach.

The unsteadiness and temporal variation of the wake during a cycle from $\beta=+10^{\circ}$ to $\beta=-10^{\circ}$ and then from $\beta=-10^{\circ}$ to $\beta$ $=+10^{\circ}$ are described in Figure 11 for several yaw angles. When examining these results, it must be taken into account that the

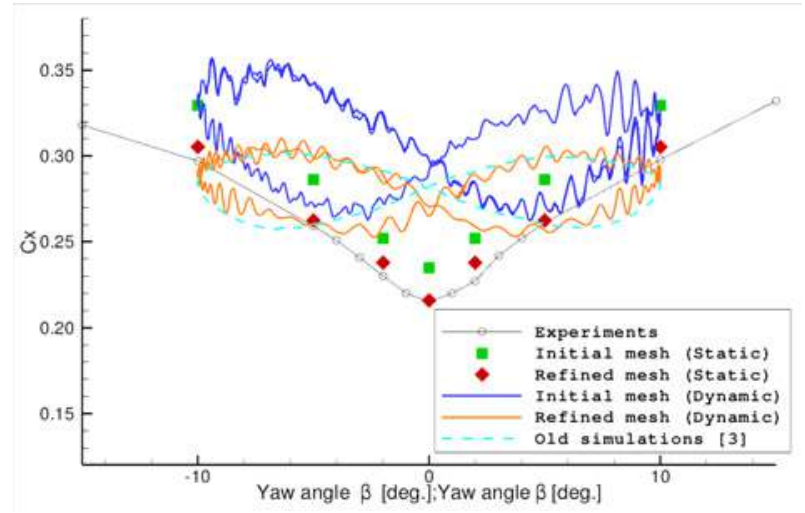

(a) DRAG COEFFICIENT.

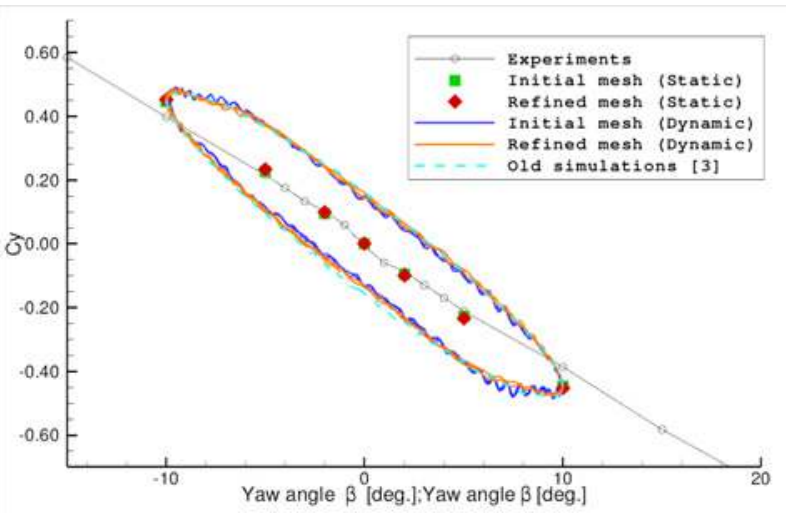

(b) SIDE FORCE COEFFICIENT.

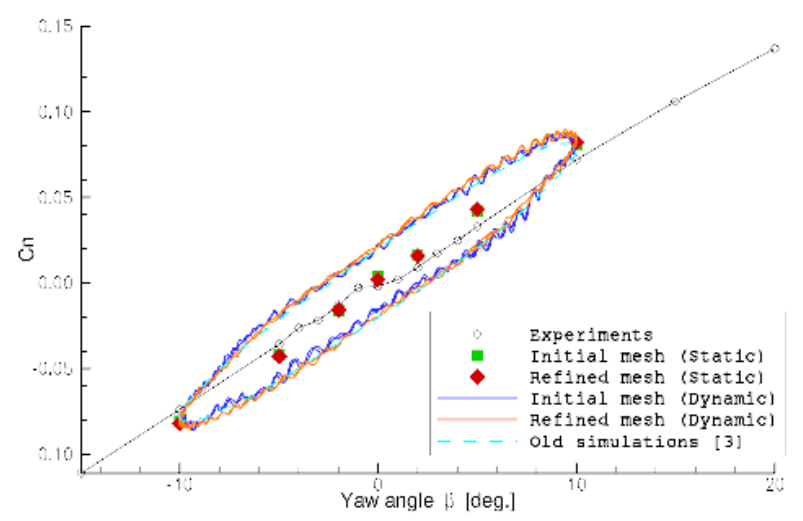

(c) YAWING MOMENT.

FIGURE 9. FORCE AND MOMENT COEFFICIENTS.

vortices which appear at $\mathrm{X} / \mathrm{L}=0.70$ are shed at the level of the base located at $\mathrm{X} / \mathrm{L}=0.51$. In frame 11 (a) where $\beta=+9.59^{\circ}$, the right side of the model is to windward and the model is moving from the yaw angle $\beta=+10^{\circ}$ to $\beta=-10^{\circ}$. The pair of vortices observed in frame 11(a) is identical to the pair of vortices shed by a square-back body placed in a cross flow in a steady wind. 


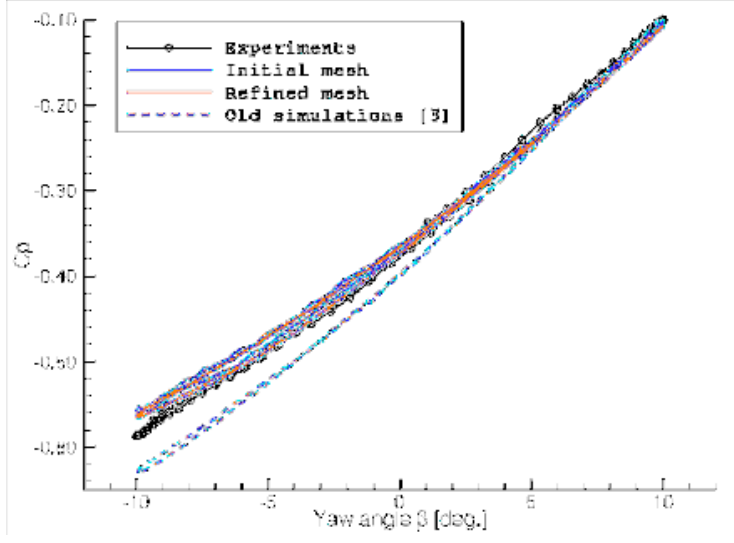

(a) Pressure tap P1

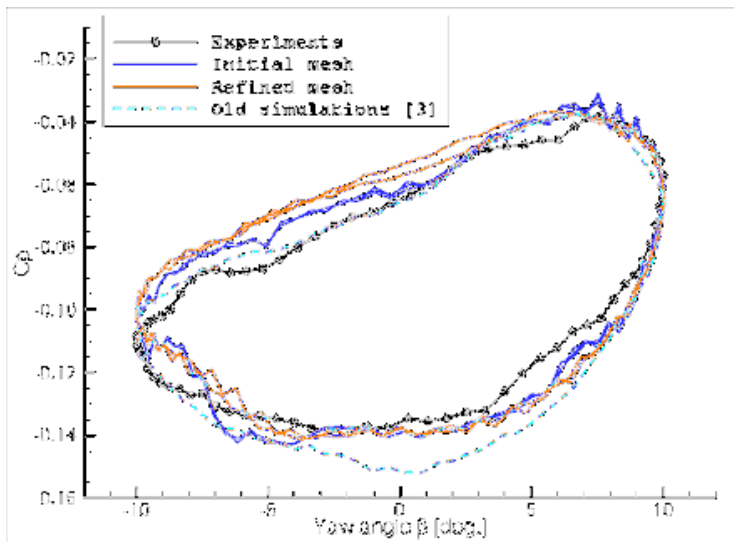

(b) Pressure tap P2

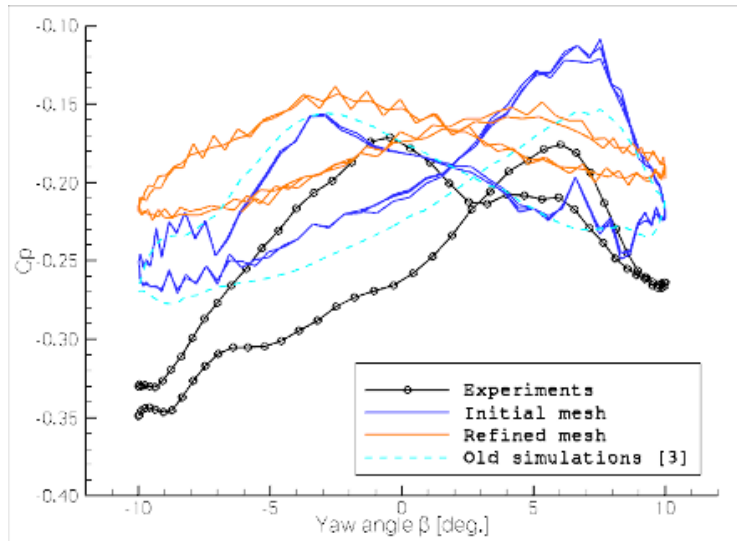

(c) Pressure tap P3

FIGURE 10. COMPARISON OF PRESSURE ON THE WILLY MODEL.

The following frames 11(b), 11(c), 11(d) show that this pair of vortices vanishes at the yaw angle $\beta=-5.35^{\circ}$ in frame $11(\mathrm{~d})$ and is replaced in frame $11(\mathrm{e})$ by a new pair of vortices which rotate

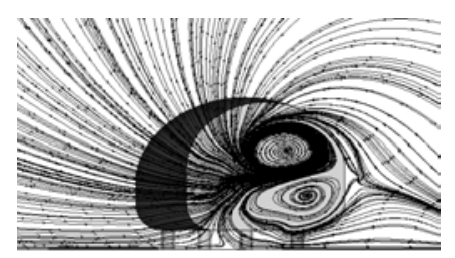

(a) $\beta=+9.59 \mathrm{deg}$.

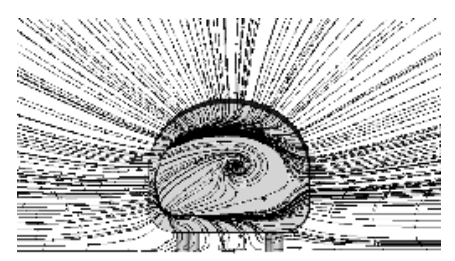

(c) $\beta=-0.08 \mathrm{deg}$.

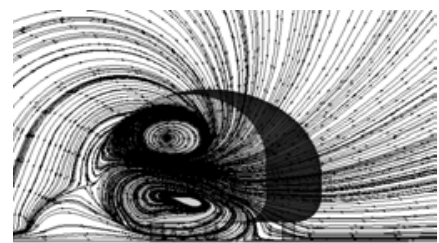

(e) $\beta=-9.61 \mathrm{deg}$.

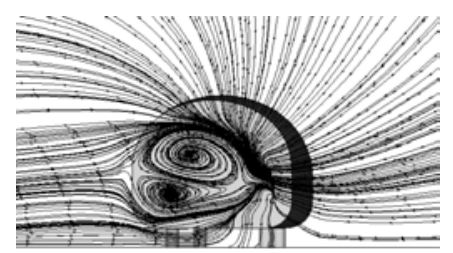

(g) $\beta=-4.84 \mathrm{deg}$

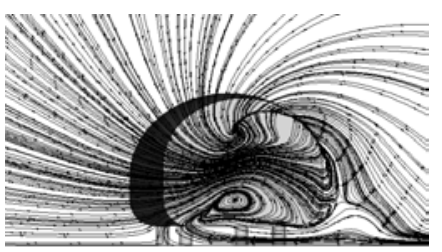

(i) $\beta=+5.32 \mathrm{deg}$.

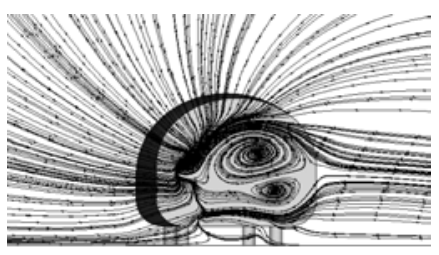

(b) $\beta=+5.35 \mathrm{deg}$.

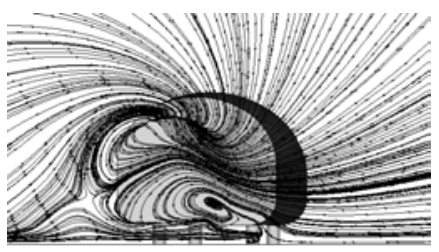

(d) $\beta=-5.35 \mathrm{deg}$.

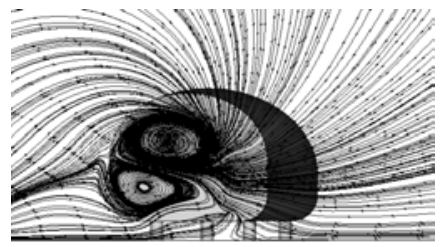

(f) $\beta=-9.47 \mathrm{deg}$.

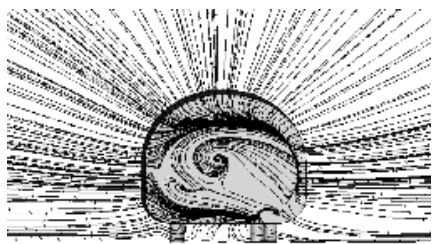

(h) $\beta=-0.07 \mathrm{deg}$.

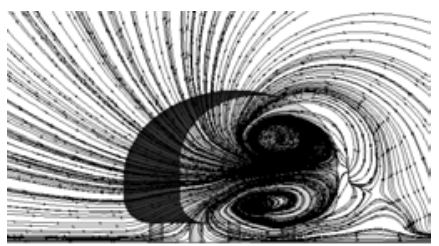

(j) $\beta=+9.55 \mathrm{deg}$.
FIGURE 11. CROSS FLOW STREAMLINES FOR ONE CYCLE AT $\mathrm{Xo} / \mathrm{L}=0.475$.

in a direction opposed to the vortices observed in frame 11(a). The physics is the same for the frames from 11(f) to 11(j), and the cross flow velocities are symmetric to the frames 11(a) to 11(e).

Fig. 12 presents isosurfaces of the non-dimensional second invariant $\mathrm{Q}^{*}=100$ for one cycle. In comparison to the previous figures, we observe also that the flow looks different for the same angle when the rotation of the body is in the direction of the 
increasing or decreasing yaw angle. For example, Figs. 11(a) and 11(j) show the flow at position $\beta=10 \mathrm{deg}$. While in Fig. 11(j) the body is finishing its rotation from $\beta=-10 \mathrm{deg}$. to $\beta=10 \mathrm{deg}$., Fig. 11(a) shows the flow when the body is rotating from $\beta=$ $10 \mathrm{deg}$. to $\beta=-10 \mathrm{deg}$. The flow does not react instantaneously to the solid body rotation of the model and it takes some time before the wake adapts to the change in the body rotation.

Figs. 13 show a comparison of the flow structures in static and in dynamic conditions for the yaw angle $\beta=0 \mathrm{deg}$. The flow in Fig. 13(a) is the result of the oscillation of the body from $\beta=$ +10 deg. to $\beta=-10$ deg. while the flow in Fig. 13(b) is the result going from $\beta=-10 \mathrm{deg}$. to $\beta=+10 \mathrm{deg}$. The result obtained in the static position at $\beta=0 \mathrm{deg}$. is presented in Fig. 13(c). These figures show that the wake for a position of the model depends

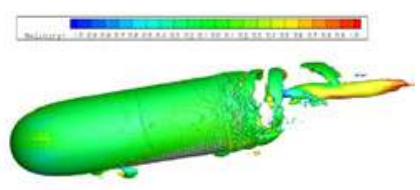

(a) $\beta=+9.59 \mathrm{deg}$.

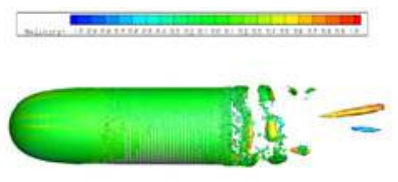

(c) $\beta=-0.08 \mathrm{deg}$.

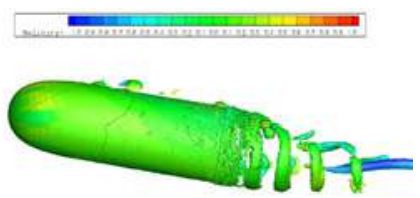

(e) $\beta=-9.61 \mathrm{deg}$.

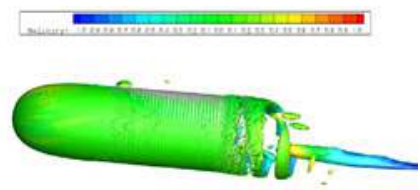

(g) $\beta=-4.84 \mathrm{deg}$.

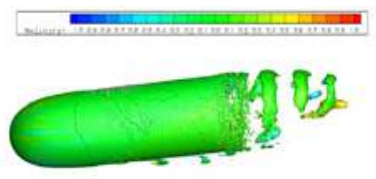

(i) $\beta=+5.32 \mathrm{deg}$.

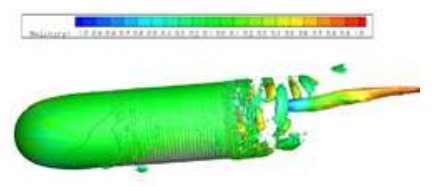

(b) $\beta=+5.35 \mathrm{deg}$.

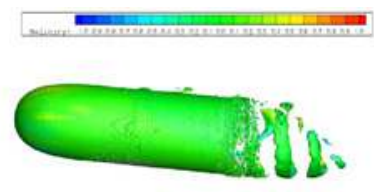

(d) $\beta=-5.35 \mathrm{deg}$.

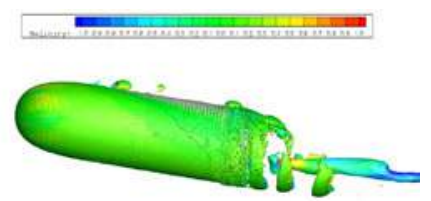

(f) $\beta=-9.47 \mathrm{deg}$.

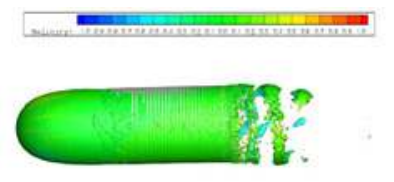

(h) $\beta=-0.07 \mathrm{deg}$.

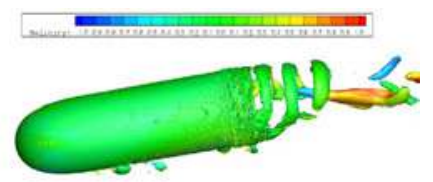

(j) $\beta=+9.55 \mathrm{deg}$.
FIGURE 12. ISOSURFACE OF THE SECOND INVARIANT $\mathrm{Q}^{*}=$ 100 FOR ONE CYCLE.

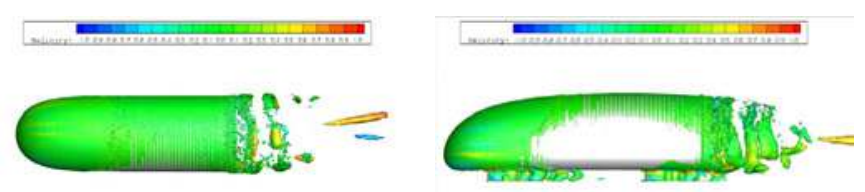

(a) FROM $\beta=+10$ DEG. TO $\beta=-10$ DEG.
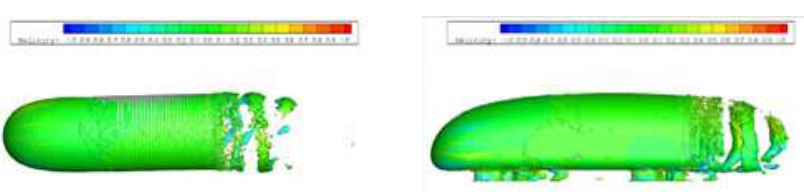

(b) FROM $\beta=-10$ DEG. TO $\beta=+10$ DEG.
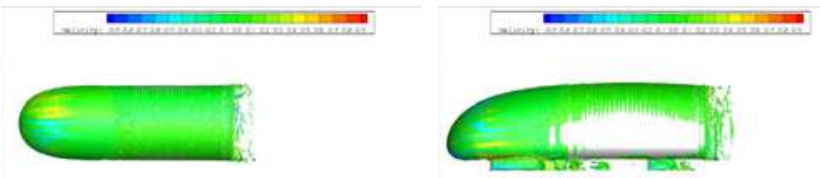

(c) STATIC.

FIGURE 13. ISOSURFACE OF THE SECOND INVARIANT Q* $=$ 100 FOR $\beta=0$ deg. (AT LEFT TOP VIEW, AT RIGHT SIDE VIEW).

on the type of simulation. In the case of dynamic position, the effect of the body rotation is not limited to the wake even at zero yaw angle. The flow remembers that the body was yawed when it arrives at this position.

\section{CONCLUSIONS}

Numerical simulations of an oscillating model were performed to explore the difference between the quasi-steady and dynamic flow conditions when used for an evaluation of the cross-wind properties of vehicles.

To obtain these results, an automatic grid refinement method has been applied. The numerical results show that without any local adaptation method of grids the drag coefficient is overestimated.

The results presented in this paper confirm that the memory effects of the flow and the inertia of the flow are important.

To take into account the experimental setup, a sliding grid approach has been used. This leads to new boundary conditions where one interpolation procedure is used which carried out to fluctuations. The reason for this increase in fluctuations is the change of connection to the neighbouring cells of the sliding interface. This change occurs from time to time between the rotating cells and the fixed cells. Even if the interpolation is second order, it seems as if the grid is changing between two time steps when a connection changes between these two time steps. One possible option is to have a smooth in time and still accurate interpolation of the neighbouring cells independent of the 
mesh. This has already been implemented for the computation of the first and second order derivatives of the pressure for adaptive grid refinement [17], on the basis of third-order least-squares approximations.

\section{ACKNOWLEDGMENT}

This work was granted access to HPC servers of CINES and IDRIS under the allocation 2011-20129 made by GENCI Grand Equipement National de Calcul Intensif) which is gratefully acknowledged.

\section{REFERENCES}

[1] Hucho, W. H., 1998. Aerodynamics of road vehicles. SAE International.

[2] Garry, K. P., and Cooper, K. R., 1986. "Comparison of quasi-static and dynamic wind tunnel measurements on simplified tractor-trailer models". Journal of Wind Engineering and Industrial Aerodynamics, 22, pp. 185-194.

[3] Guilmineau, E., and Chometon, F., 2008. Numerical and experimental analysis of unsteady separated flow behind an oscillating car model. SAE Paper 2008-01-0738.

[4] Krajnović, S., Bengtsson, A., and Basara, B., 2011. "Large Eddy Simulation Investigation of the Hysteresis Effects in the Flow Around an Oscillating Ground Vehicle". Journal of Fluids Engineering, 133. 121103.

[5] Guilmineau, E., and Chometon, F., 2009. "Effect of side wind on a simplified car model: Experimental and numerical analysis". Journal of Fluids Engineering, 131. 021104.

[6] Watkins, S., and Saunders, J. W., 1995. Turbulence experienced by road vehicles under normal driving conditions. SAE Paper 950997.

[7] Menter, F. R., 1993. "Zonal two-equation $k-\omega$ turbulence models for aerodynamic flows". In AIAA 24th Fluid Dynamics Conference, AIAA Paper 93-2906.

[8] Deng, G. B., Queutey, P., and Visonneau, M., 2005. “Threedimensional flow computation with reynolds stress and algebraic stress models". In Engineering Turbulence Modelling and Experiments 6, W. Rodi and M. Mulas, eds., Proceedings of the ERCOFTAC International Symposium on Engineering Turbulence Modelling and Measurements ETMM6, Elsevier, pp. 389-398.

[9] Duvigneau, R., Visonneau, M., and Deng, G. B., 2003. “On the role played by turbulence closures in hull ship optimization at model and full scale". Journal of Marine Science and Technology, 8, pp. 11-25.

[10] Leroyer, A., Queutey, P., and Visonneau, M., 2007. "Couplage coulement/mouvement au sein d'un solveur RANSE : Application à l'hydrodynamique navale". In 11 ième Journées de l'Hydrodynamique, pp. 61-74.
[11] Rhie, C., and Chow, W., 1983. "A numerical study of the turbulent flow past an isolated aerofoil with trailing edge separation". AIAA Journal, 17, pp. 1525-1532.

[12] Blades, E. L., and Marcum, D. L., 2007. "A sliding interface method for unstructured flow simulations". International Journal for Numerical Methods in Fluids,, 53(9), pp. 507-529.

[13] Kim, H.-G., 2008. "Development of three-dimensional interface elements for coupling of non-matching hexahedral meshes". Computer Methods in Applied Mechanics and Engineering, 197(45-48), pp. 3870-3882.

[14] George, P. I., and Borouchaki, H., 1998. Delaunay triangulation and meshing: Application to finite element. Hermes.

[15] Alauzet, F., and Loseille, A., 2010. "High-order sonic boom modeling based on adaptive methods". Journal of Computational Physics, 229(3), pp. 561-593.

[16] Wackers, J., Said, K. A., Queutey, P., and Visonneau, M., 2010. "Adaptive grid refinement applied to RANS ship flow computation". In 28th Symposium on Naval Hydrodynamics.

[17] Wackers, J., Deng, G. B., Leroyer, A., Queutey, P., and Visonneau, M., 2012. "Adaptive grid refinement for hydrodynamic flow simulation". Computers \& Fluids, 55, pp. 85100.

[18] Deng, G. D., and Visonneau, M., 1999. "Comparison of explicit algebraic stress models and second-order turbulence closures for steady flow around ships". In 7th Symposium on Numerical Ship Hydrodynamics, pp. 4.4-1-4.4-15.

[19] Guilmineau, E., Chickahoui, C., Deng, G. B., and Visonneau, M., 2011. "Cross wind effects on a simplified car model by a DES approach". Computers \& Fluids. doi:10.1016/j.compfluid.2011.08.02. 